\title{
Host-microbe interactions in the gut of Drosophila melanogaster
}

\author{
Takayuki Kuraishi ${ }^{1,2+}$, Aki Hori ${ }^{1+}$ and Shoichiro Kurata ${ }^{1 *}$ \\ ${ }^{1}$ Department of Molrcular Biopharmacy and Genetics, Graduate School of Pharmaceutical Sciences, Tohoku University, Sendai, Japan \\ 2 PRESTO, Japan Science and Technology Agency, Tokyo, Japan
}

\author{
Edited by: \\ Shinji Nagata, University of Tokyo, \\ Japan \\ Reviewed by: \\ Sara Cherry, University of \\ Pennsylvania, USA \\ Shun-ichiro Kawabata, Kyushu \\ University, Japan \\ *Correspondence: \\ Shoichiro Kurata, Department of \\ Molrcular Biopharmacy and \\ Genetics, Graduate School of \\ Pharmaceutical Sciences, Tohoku \\ University, Aoba 6-3, Aramaki, \\ Aoba-ku, Sendai 980-8578, Japan \\ e-mail:kurata@m.tohoku.ac.jp \\ tThese authors have contributed \\ equally to this work.
}

Many insect species subsist on decaying and contaminated matter and are thus exposed to large quantities of microorganisms. To control beneficial commensals and combat infectious pathogens, insects must be armed with efficient systems for microbial recognition, signaling pathways, and effector molecules. The molecular mechanisms regulating these host-microbe interactions in insects have been largely clarified in Drosophila melanogaster with its powerful genetic and genomic tools. Here we review recent advances in this field, focusing mainly on the relationships between microbes and epithelial cells in the intestinal tract where the host exposure to the external environment is most frequent.

Keywords: Drosophila, innate immunity, peritrophic matrix, antimicrobial peptide, reactive oxygen species, intestinal stem cell, gut flora

\section{INTRODUCTION}

Microorganisms exist in all parts of the biosphere, which exerts pressure on multicellular organisms to develop mechanisms to effectively respond to microbes. In humans, for example, as much as $2 \mathrm{~kg}$ of indigenous bacteria are thought to be contained in the intestine, the respiratory tract, the genitals, and on the skin, an amount that corresponds to 10 times the number of host cells. The gut epithelium, the body part frequently exposed to the external environment, contains the majority of the commensals in the body. Intestinal bacteria benefit the host by gleaning energy from the fermentation of undigested carbohydrates and facilitating the subsequent absorption of short chain fatty acids by the host. They also play a role in synthesizing vitamins and in metabolizing bile acids and sterols (Cummings and MacFarlane, 1997). Recent studies revealed that microbiota also contribute to maintain host homeostasis and immune responses. Deregulated alterations of microbiota induce chronic inflammation in the intestine (Sartor, 2004; Round and Mazmanian, 2009), as well as cancer in the liver (Yoshimoto et al., 2013).

Because the gut epithelium is frequently exposed to harmful pathogens, it must be armed with an efficient and powerful immune system to protect itself. The fruit fly Drosophila melanogaster possesses a gut that is structurally and functionally similar to mammalian intestinal tract (Lemaitre and MiguelAliaga, 2013), which is constantly in contact with microbial pathogens as flies ingest large quantities of microorganisms through feeding on rotting fruits. Drosophila is a powerful model organism for deciphering innate immune responses (Lemaitre and Hoffmann, 2007). Analysis of systemic immune responses of Drosophila in response to the direct invasion of pathogens into the body fluid revealed that the Toll pathway is important for fungal infection and led to the discovery of the mammalian Toll-like receptor (TLR) signaling pathway (O'Neill et al., 2013). TLR signaling is crucial to the first line of defense against pathogens as well as the induction of adaptive immunity (Iwasaki and Medzhitov, 2010; Takeuchi and Akira, 2010). Recent studies in Drosophila have begun to reveal the mechanisms that regulate gut defense against bacterial infection and provide insights into the mammalian intestinal defense system (Royet, 2011; Buchon et al., 2013a; Ferrandon, 2013). Drosophila gut defense responses comprise four steps: (i) physical barriers, such as the peritrophic matrix and epithelial integrity (Hegan et al., 2007; Bonnay et al., 2013), (ii) production of reactive oxygen species (ROS), (iii) secretion of antimicrobial peptides (AMPs) into the hemolymph through the Imd pathway, and (iv) epithelium renewal to maintain homeostasis in response to gut damage. In the first half of this review, we describe these four steps in more detail.

On the other hand, Drosophila also possess indigenous gut microbiota that have important roles in the host physiology and pathology (Broderick and Lemaitre, 2012). How flies discriminate benign and beneficial bacteria from pathogenic microbes to maintain harmonious gut flora, however, has remained unclear. In the latter part of this review, we describe the importance of the ROS-producing pathway and negative regulators of the Imd pathway for maintaining commensals, and discuss possible future directions of the gut immunology.

\section{PERITROPHIC MATRIX AS A PHYSICAL BARRIER OF GUT EPITHELIUM}

The peritrophic matrix is an acellular structure that forms a layer comprising chitin polymers and glycoproteins, such as peritrophins, lining the insect midgut lumen (Figure 1B) (Lehane, 1997; Hegedus et al., 2009). The peritrophic matrix, though structurally different, is considered analogous to the mucus layer of 


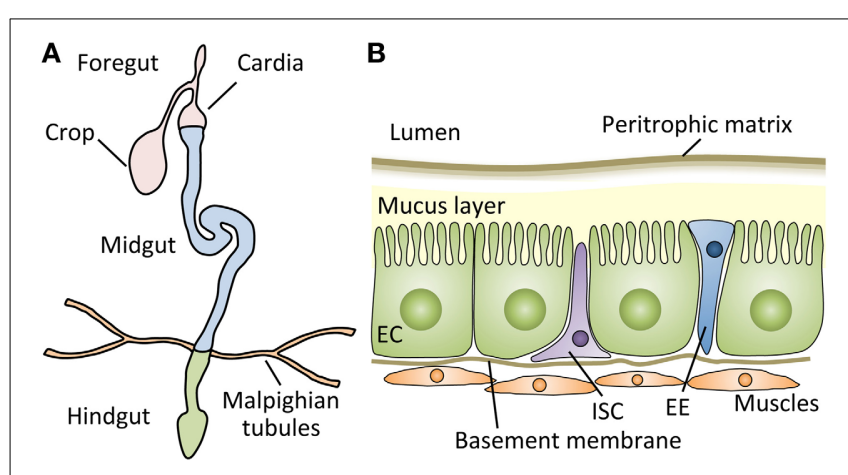

FIGURE 1 | The structure of the Drosophila gut. (A) Schematic representation of the digestive tract of the Drosophila adult. The Drosophila gut is a tubular epithelial organ composed of a monolayer of cells surrounded by muscles. The gut is divided into the foregut, the midgut, and the hindgut, based on the developmental origin. The crop stores the food ingested by the flies, and the first half of the cardia belongs to the foregut. The Malpighian tubules, a functional equivalent of the mammalian kidney, connect at the midgut-hindgut junction. The midgut is the main site of digestion, and most studies of Drosophila gut immunity have focused on this compartment. (B) A cross section of the adult Drosophila midgut. The adult midgut contains several types of cells: absorptive enterocytes (ECs), secretory enteroendocrine cells (EEs), and pluripotent intestinal stem cells (ISCS). Muscle cells are present under the basement membrane of epithelial cells. Between the lumen and epithelia, a semipermeable non-cellular structure, the peritrophic matrix, protects the enterocytes from abrasive particles and pathogens. In addition, a mucus layer lies between the peritrophic matrix and ECs along the midgut (Ridley et al., 2012).

the mammalian digestive tract, and shields the midgut epithelium from abrasive food particles and microbes (Hegedus et al., 2009). Indeed, studies in insects suggest that the peritrophic matrix protects hosts from xenobiotics and toxins, such as dichlorodiphenyl-trichloroethane (DDT) and Bacillus thuringiensis (Bt) toxins (Tellam, 1996; Hayakawa et al., 2004). Interestingly, Plasmodium species secrete chitinases that disrupt the peritrophic matrix, which facilitates their passage through the midgut epithelia in mosquitos (Abraham and Jacobs-Lorena, 2004).

Drosocrystallin (Dcy) protein is a recently identified component of the peritrophic matrix in the adult Drosophila midgut, and genetic evidence suggests a protective role of the peritrophic matrix against pathogens (Kuraishi et al., 2011). dcy encodes a chitin-binding protein and its expression is upregulated upon the ingestion of bacteria. A strong loss-of-function mutation in $d c y$ reduces the peritrophic matrix width by half and increases its permeability to larger molecules, suggesting that, despite the high number of structural proteins associated with the peritrophic matrix, Dcy is an essential component of the peritrophic matrix in the Drosophila adult. Moreover, $d c y$-deficient flies exhibit increased susceptibility to oral infections by the entomopathogenic bacteria Pseudomonas entomophila (Vodovar et al., 2005) and Serratia marcescens. dcy mutant flies also succumb faster than wild-type flies upon ingestion of a $P$. entomophila extract. This lethality is due in part to Monalysin (Opota et al., 2011; Blemont et al., 2013), a pore-forming toxin produced by P. entomophila. Furthermore, the ingestion of bacteria induces a higher level of expression of AMPs in the dcy mutant (Kuraishi et al., 2011), implying that the peritrophic matrix modulates immune responses in the gut.
Though the precise mechanism underlying transcriptional control of the $d c y$ gene remains to be determined, microarray analysis comparing the transcriptome following ingestion of non-lethal Erwinia carotovora with that of lethal P. entomophila revealed that $d c y$ induction upon the ingestion of the lethal bacteria is approximately four times higher than that of the non-lethal bacteria (Chakrabarti et al., 2012), suggesting that dcy expression is regulated by the degree of stress or damage to the gut.

The peritrophic matrix is a semipermeable membrane, and its permeability is partly controlled by protein-protein crosslinking by transglutaminase in Drosophila (Shibata et al., 2013). Along this line, in the mosquito Anopheles gambiae, the mucus layer, which is cross-linked by dityrosine covalent bonds, and the peritrophic matrix, control gut permeability. Peroxidase/dual oxidase (Duox) forms this dityrosine network to decrease gut permeability to immune elicitors from Plasmodium, thereby preventing the activation of immune responses in the gut (Kumar et al., 2010). Curiously, $d c y$ is exclusively expressed in the adult stage, though Drosophila larvae also have a peritrophic matrix, and no $d c y$ homolog has been identified outside the Drosophilidae family. The extreme diversity of insect species in terms of their mode of life and way of feeding, leads them to encounter different kinds of microbes and abrasive particles. Distinct compositions of the peritrophic matrix and dynamic modifications of its permeability by protein-protein cross-linking in each insect species or at different life stages might allow them to adjust to their various environments by ensuring a protective role of the peritrophic matrix against invaders.

\section{PRODUCTION OF REACTIVE OXYGEN SPECIES FOLLOWING ORAL INFECTION}

NADPH oxidase family proteins are membrane-bound enzymes that catalyze the generation of ROS. They are localized in the plasma membrane as well as in the phagosomal membrane. NADPH oxidases in phagosomes kill engulfed bacteria in neutrophils and macrophages (Babior, 1999). Duox is an enzyme belonging to the NADPH oxidase family proteins in Drosophila, and is likely to be expressed in the plasma membrane. Duox is an important factor responsible for gut defense (Ha et al., 2005; Bae et al., 2010). In fact, Duox-RNAi flies are susceptible to the ingestion of yeast and several bacterial species.

In laboratories, Drosophila is reared with yeast-supplemented food, thus the fly is considered to be in a slightly infected condition at all times. Upon routine ingestion of dietary yeasts, phospholipase $C \beta$ (PLC $\beta$ ) in the gut epithelial cells is activated through the $G$ protein alpha subunit $q$ protein $(G \alpha q)$, which then mobilizes intracellular $\mathrm{Ca}^{2+}$ through inositol $(1,4,5)$ triphosphate generation. This leads to the enzymatic activation of Duox (Ha et al., 2009a) (Figure 2). Upon severe infection with a heavy microbial burden, the host must produce much more ROS to eliminate pathogens. In this case, Duox expression is increased through triggering the MEKK1/MEK3/p38 pathway, which activates Activating Transcription Factor 2 (ATF2) and eventually induces Duox transcription (Ha et al., 2009b). This MAPK pathway is important for eliminating pathogenic bacteria from the gut. 


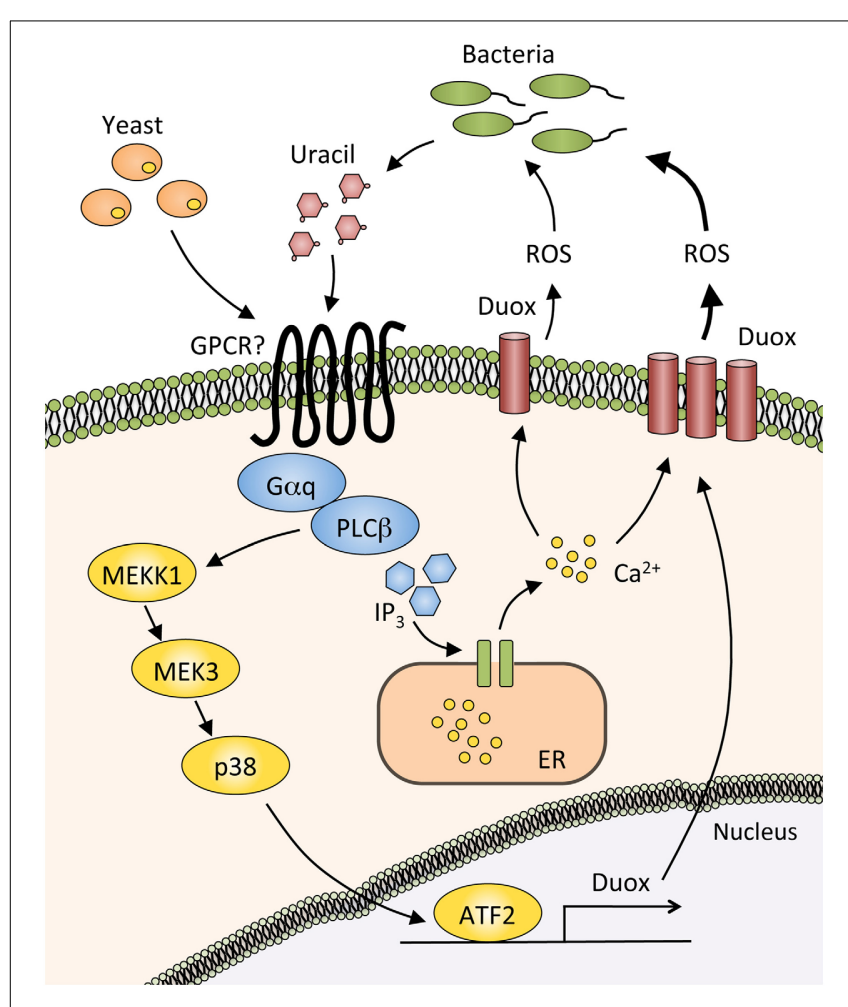

FIGURE 2 | Production of reactive oxygen species by the Duox pathway. In basal conditions, dietary yeasts or small amount of uracil from commensal bacteria activate the $\mathrm{G}$ protein alpha subunit $\mathrm{q}(\mathrm{G} \alpha \mathrm{q})$ and phospholipase $\mathrm{C} \beta(\mathrm{PLC} \beta)$, thereby inducing the synthesis of inositol $(1,4,5)$-triphosphate $\left(\mathrm{IP}_{3}\right)$, which binds to $\mathrm{IP}_{3}$ receptor in the endoplasmic reticulum (ER). Calcium ions are then released from ER into the cytosol, resulting in the activation of a reactive oxygen species (ROS)-producing enzyme Dual oxidase (Duox). Duox expression is strongly induced by the large amount of uracil produced by pathogenic bacteria. PLC $\beta$ mediates this Duox upregulation through triggering the MEKK1/MEK3/p38 MAPK pathway and activating Activating Transcription Factor2 (ATF2).

A recent study revealed that bacterial-derived uracil mediates Duox-dependent ROS production in Drosophila gut (Lee et al., 2013). Uracil is released by pathogenic bacteria such as E. carotovora and Gluconobacter morbifer, and possibly activates an as-yet unidentified $G$ protein-coupled receptor that would be upstream of the G $\alpha$ q-PLC $\beta$. Symbiotic microbes such as Lactobacillus plantarum and Acetobacter pomorum have lower ROS-producing effects, probably because those commensals release less uracil. These findings suggest that uracil released by bacteria acts as a factor that classifies microbes as symbiotic or pathogenic.

Although ROS is an important factor for gut homeostasis in Drosophila, it damages the gut epithelia upon infection, leading the host to repair this injury by the proliferation and differentiation of intestinal stem cells (ISCs).

\section{RELEASE OF ANTIMICROBIAL PEPTIDES IN THE GUT}

AMPs are evolutionarily conserved peptides that can damage and kill microbes, and are found among all classes of life (Zasloff, 2002). The production and regulation of AMPs upon infection are well characterized in Drosophila, and AMPs produced by the fat body upon systemic infection in Drosophila adults are required for host defense (Lemaitre and Hoffmann, 2007). Two major signaling pathways, the Toll and Imd pathways, control the expression of AMPs in the systemic immune response (Lemaitre and Hoffmann, 2007; Kurata, 2010).

In the gut, the Imd pathway controls the generation of AMPs (Tzou et al., 2000; Ryu et al., 2006; Buchon et al., 2009b). This gut immune response is triggered by the recognition of diaminopimelic acid-type peptidoglycan, which is derived from almost all Gram-negative bacteria and a subclass of Grampositive bacteria, by pattern recognition receptor peptidoglycan recognition protein (PGRP)-LE and PGRP-LC (Bosco-Drayon et al., 2012; Neyen et al., 2012) (Figure 3). Microarray analyses upon the ingestion of E. carotovora or P. entomophila showed that AMPs and other immune-related molecules are induced in an Imd pathway-dependent manner (Buchon et al., 2009b; Chakrabarti et al., 2012). Flies deficient for Imd pathway activation are more sensitive to oral infection with pathogenic bacteria such as P. entomophila and S. marcescens, confirming the important contribution of the Imd pathway to host defense in the gut (Liehl et al., 2006; Nehme et al., 2007).

On the other hand, pathogenic bacteria resist the action of AMPs. A genetic study with $P$. entomophila revealed that the aprA gene is necessary for the virulence of P. entomophila, and a subsequent study identified that aprA encodes a secreted metalloprotease that is likely to protect the pathogen against AMPs by deactivating them (Liehl et al., 2006). This study revealed a strategy of pathogenic bacteria to escape from host gut immune responses.

\section{GUT REPAIR AFTER DAMAGE BY ORAL INFECTION}

The gut of Drosophila is a compartmentalized organ similar to the mammalian intestinal tract (Figure 1A): i.e., its main function is the digestion and absorption of foods, it has a tubular epithelium surrounded by muscles, its activity is partly under the control of the nervous system, and its constant and rapid turnover throughout the lifespan maintain host homeostasis (Cognigni et al., 2011; Buchon et al., 2013b; Marianes and Spradling, 2013). The adult Drosophila gut possesses ISCs (Micchelli and Perrimon, 2006; Ohlstein and Spradling, 2006). Their proliferation and differentiation under healthy conditions are regulated by the molecular mechanisms involving the Delta-Notch, Wingless, and Pvr signaling pathways (Micchelli and Perrimon, 2006; Ohlstein and Spradling, 2006; Lin et al., 2008; Bond and Foley, 2012), similar to the mammalian intestine, to maintain its homeostasis. Drosophila adult ISCs self-renew and produce two main cell types of the gut: enterocytes that absorb nutrients and enteroendocrine cells that secrete enteric hormones.

Upon oral infection by bacteria, the gut epithelia are damaged and killed by ROS produced by Duox (Buchon et al., 2009a). In fact, oral infection with E. carotovora causes the loss of approximately half of the intestinal cells compared to its healthy condition, and a significant shortening of the gut is observed after infection (Buchon et al., 2010). To repair the damage induced by oral infection and maintain gut homeostasis, ISCs are activated to proliferate and differentiate into new enterocytes. The JAK-STAT, 


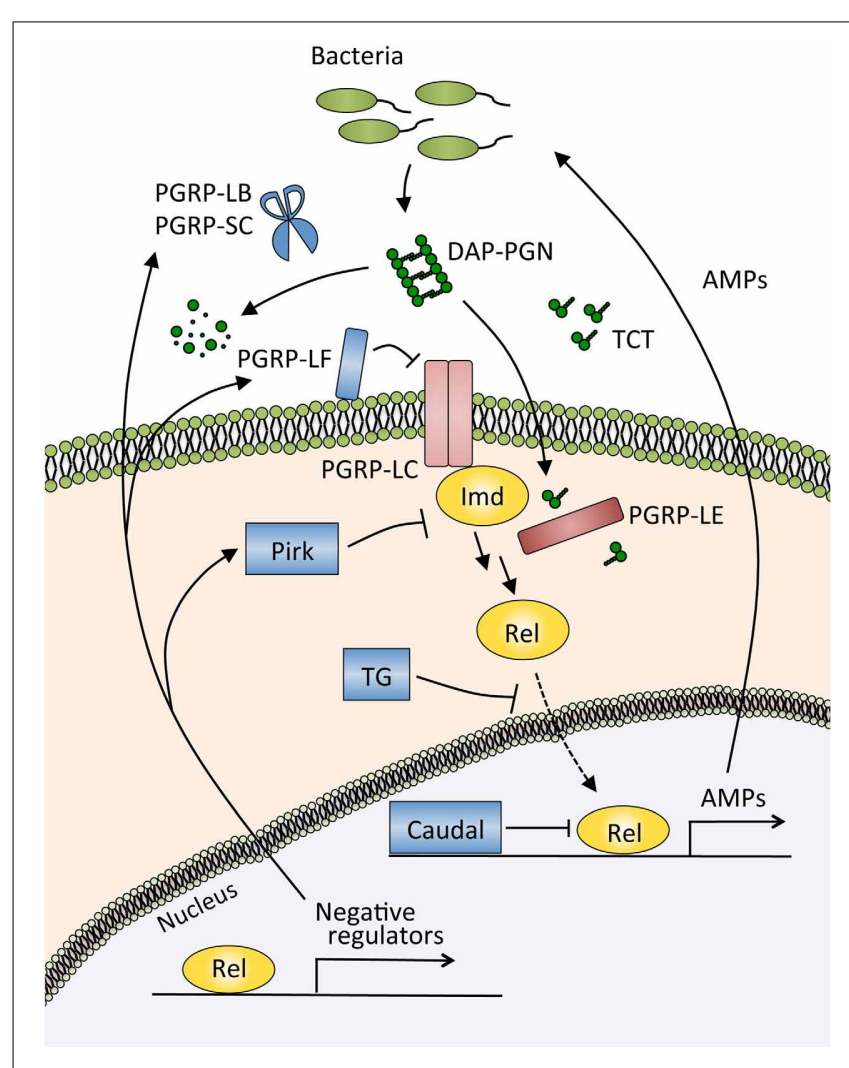

FIGURE 3 | The Imd pathway and its negative regulators in the gut. The Imd pathway is activated by the recognition of diaminopimelic acid-type peptidoglycan (DAP-PGN) by the membrane receptor PGRP-LC, or tracheal cytotoxin (TCT) by the intracellular receptor PGRP-LE. Activated PGRP-LC or PGRP-LE then recruits the adaptor protein Imd, which triggers the signaling cascade of the pathway and induces the nuclear translocation of Relish, a nuclear factor-kB (NF-kB) transcription factor of Drosophila. The NF-kB protein Relish activates the expression of antimicrobial peptides (AMPs) and negative regulators of this pathway. PGRP-LB and PGRP-SC catalyze the degradation of peptidoglycan and TCT, resulting in a decrease in the amount of ligands for the recognition receptors PGRP-LC and PGRP-LE. PGRP-LF and Pirk inhibit the activity of those receptors, transglutaminase (TG) blocks the nuclear translocation of Relish, and Caudal modulates the transcriptional response of Relish. epidermal growth factor receptor (EGFR), Hippo and Wingless pathways in the ISCs are required for ISC proliferation and differentiation (Apidianakis et al., 2009; Buchon et al., 2009a,b, 2010; Chatterjee and Ip, 2009; Cronin et al., 2009; Jiang et al., 2009, 2011; Cordero et al., 2012; Zhou et al., 2013) (Figure 4). The JNK and Hippo pathways are activated in damaged enterocytes upon infection (Jiang et al., 2009; Karpowicz et al., 2010; Shaw et al., 2010; Staley and Irvine, 2010; Bond and Foley, 2012). Those enterocytes produce a secreted cytokine, Upd3, a ligand for the receptor Domeless, which activates the JAK-STAT pathway in ISCs to promote both their division and differentiation into enterocytes. Upd3 also acts in visceral muscles to direct the production of an epidermal growth factor, Vein, which triggers the EGFR/Ras/MAPK pathway in ISCs to promote their proliferation. Vein also acts in enterocytes to properly coordinate the exclusion of damaged cells (Buchon et al., 2010; Biteau and Jasper,

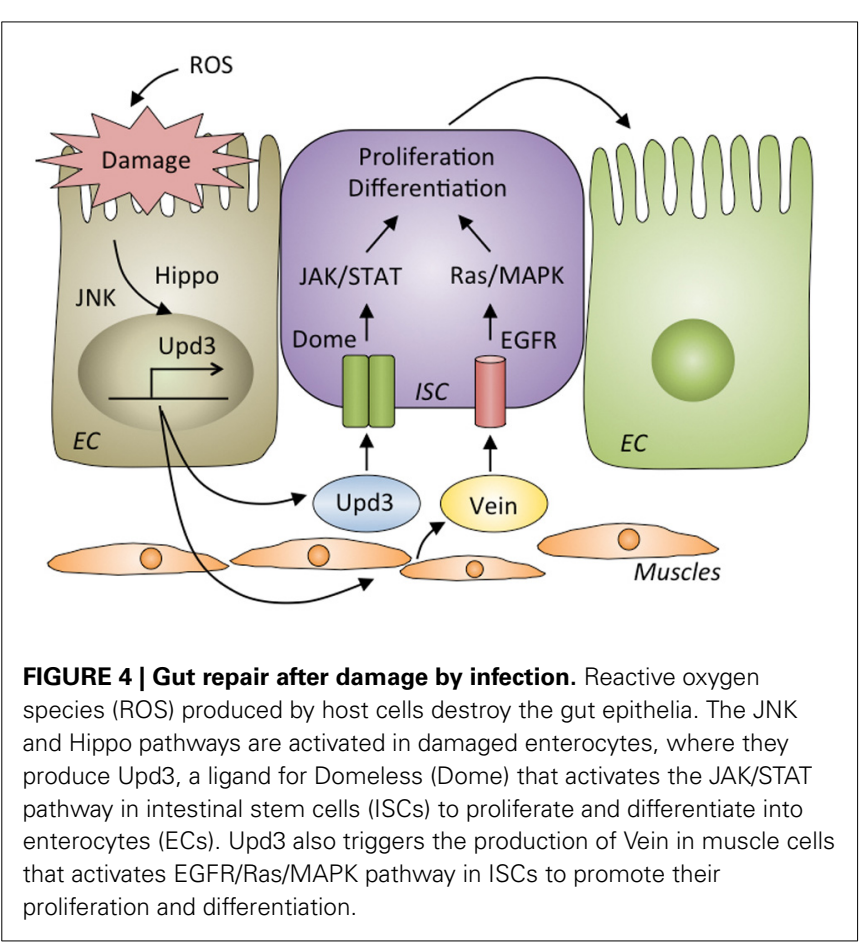

2011; Jiang et al., 2011; Zhou et al., 2013). The JAK-STAT and EGFR pathways in ISCs and the EGFR pathway in enterocytes are indispensable for maintaining homeostasis, as flies lacking those pathways in the corresponding cells are highly susceptible to infection (Buchon et al., 2009a, 2010; Jiang et al., 2009; Osman et al., 2012).

Severe infection with the lethal pathogen $P$. entomophila blocks these gut repair pathways. Excessive ROS and poreforming toxins from P. entomophila modulate stress pathways, specifically activation of the GCN2 kinase and inhibition of the target of rapamycin (TOR) pathway, leading to a global translational blockage (Chakrabarti et al., 2012). Consequently, repair cytokines such as Upd3 and Vein are not secreted upon severe infection, leading to failed epithelium renewal and resulting in the death of the fly.

\section{COMMENSAL FLORA OF THE Drosophila GUT}

The Drosophila gut, like the mammalian intestinal tract, is associated with a number of microorganisms, though the bacterial diversity of flies ( $\sim 30$ different species) is lower than that of mammals (>500 species) (Broderick and Lemaitre, 2012). The most commonly associated bacterial species in flies are members of the Lactobacillus and Acetobacter groups (Wong et al., 2011). These bacteria have an important role in host physiology, specifically larval growth (Ridley et al., 2012).

Germ-free larvae reared in conditions of nutrient scarcity exhibit delayed development (Storelli et al., 2011). This effect is mediated by L. plantarum, as re-introduction of this bacterium into germ-free larvae is sufficient to restore the natural growth rate. L. plantarum impacts the host TOR pathway, a major sensor of the nutritional status of the cell, and increases the release of insulin-like peptides into the larval hemolymph. 
A. pomorum is a member of the gut microbiota that affects larval size and development time under conditions of proteinpoor diets (Shin et al., 2011). Bacterial genetic studies revealed that the pyrroloquinoline quinone-dependent alcohol dehydrogenase (PQQ-ADH)-dependent oxidative respiratory chain of A. pomorum mediates this effect. Indeed, re-introduction of the PQQ-ADH-deficient A. pomorum into the larvae and supplementing the food with acetic acid, a metabolic product of the action of PQQ-ADH, rescues the growth rate of the germ-free larvae.

Gut microbiota likely affect Drosophila gut immune responses. Drosophila larvae devoid of commensal bacteria are more susceptible than wild-type larvae to infection with Candida albicans, suggesting a role of the commensals in host defense (Glittenberg et al., 2011). The molecular mechanism underlying this effect, however, is unclear.

\section{NEGATIVE REGULATORS OF THE Imd PATHWAY}

As discussed above, by regulating ROS production, bacterialderived uracil is a factor that could explain how flies maintain benign and beneficial bacteria in the gut. In addition, so-called "negative regulators" of the Imd pathway ensure an appropriate level of immune responses in the gut to establish immune tolerance against commensal microorganisms (Lee and Ferrandon, 2011; Kleino and Silverman, 2013). Some of these negative regulators are upregulated by activation of the Imd pathway, establishing a negative feedback loop that adjusts the magnitude of AMP production in the gut.

PGRPs are evolutionally conserved proteins involved in the recognition and degradation of peptidoglycans, a cell wall component of bacteria (Kurata, 2004). There are 13 PGRP family members in the Drosophila genome, and 4 family members in humans. PGRP-LB and PGRP-SC, both of which belong to the amidase PGRP family that catalyzes the degradation of peptidoglycan, reduce the levels of Imd pathway-activating ligands in the gut (Bischoff et al., 2006; Zaidman-Rémy et al., 2006; Paredes et al., 2011). Other types of negative regulators directly suppress the activity of the PGRP receptors in the Imd pathway (Figure 3). Membrane-associated protein PGRP-LF probably blocks the dimerization of PGRP-LC, and an intracellular protein Pirk interferes with the interaction of PGRP-LC or PGRP-LE with Imd, thus limiting the activation of the Imd pathway (Aggarwal et al., 2008; Kleino et al., 2008; Lhocine et al., 2008; Maillet et al., 2008; Basbous et al., 2011). The nuclear factor- $\kappa \mathrm{B}(\mathrm{NF}-\kappa \mathrm{B})$ protein Relish is also controlled by negative regulators. The homeobox protein Caudal, expressed in the posterior midgut, downregulates the expression of AMPs (Ryu et al., 2008). Transglutaminase catalyzes the proteinprotein cross-linking of Relish, and the cross-linked Relish has diminished ability to translocate to the nucleus (Shibata et al., 2013).

Flies lacking these negative regulators of the Imd pathway have a shorter lifespan, probably because of the continuous activation of the Imd pathway by the gut microbiota (Paredes et al., 2011). Interestingly, wild-type flies that ingest gut lysates prepared from transglutaminase-RNAi flies also have a shorter lifespan (Shibata et al., 2013), suggesting that maintaining the proper gut commensals is crucial to fly survival and that the Imd pathway has an important role in shaping healthy gut commensals.

\section{PERSPECTIVES}

Recent studies of the gut immune responses in Drosophila revealed resistance and tolerance mechanisms (Ayres and Schneider, 2012) against pathogens: ROS and AMPs kill invading bacteria, the peritrophic matrix diminishes the action of bacterial toxins, and epithelial renewal ensures gut homeostasis after infection-induced damage. To date, most studies have been performed by using Gram-negative bacteria as the pathogens, and thus the mechanisms of coping with oral infection by Grampositive bacteria, fungi, viruses, and protozoa remain to be clarified. With regard to systemic immune responses, the Toll pathway is required for the defense against Gram-positive bacteria (Lemaitre and Hoffmann, 2007). On the other hand, because of the acidic conditions of the gut and the presence of digestive enzymes, the proteolytic cascades that produce the Toll ligand Spätzle are likely to be inactive in the gut. Thus, Toll signaling does not seem to have a role in gut immunity (Buchon et al., 2009b), suggesting that other effector mechanisms are involved in the gut immune response against Gram-positive bacteria and fungi. Analogously, as a systemic immune response, hemocytes are involved in an encapsulation reaction against parasites intruding into the hemolymph (Lemaitre and Hoffmann, 2007). The observation that hemocytes are likely to be absent in the adult gut lumen implies the existence of distinct defense mechanisms against protozoa in the gut immunity. Gram-positive bacteria Enterococcus faecalis and Enterococcus faecium cause a gastrointestinal infectious disease in the silkworm. Malaria parasites and dengue viruses invade mosquitos through their gut epithelium (Cirimotich et al., 2010; Lambrechts et al., 2010; Yassine and Osta, 2010). Therefore, deciphering the immune responses to those pathogens in Drosophila would be useful for application to other insects of economic or global health importance.

Research of Drosophila gut immunity is likely relevant not only to other insects but also to mammals. Recent reports revealed that PGRPs and AMPs in mice significantly contribute to controlling gut microbiota and thereby maintaining intestinal homeostasis. There are four PGRPs in the mouse genome, and mice lacking all these PGRPs are more susceptible than wild-type mice to dextran sulfate sodium (DSS)-induced colitis, which comprises a severe loss of epithelial cells and colon ulceration, and seems to result from the proliferation of more inflammatory gut microflora upon exposure to the DSS (Saha et al., 2010). Similarly, AMPs are important for maintaining healthy microbiota in mice. Angiotensin I converting enzyme (peptidyldipeptidase A) 2 (ACE2), with its the renin-angiotensin systemindependent function, regulates the homeostasis of dietary amino acid tryptophan, which modulates the expression level of AMPs in the intestine. ACE2 knockout mice exhibit alterations in AMP expression, which probably affects the ecology of gut microbiota, ultimately leading to the susceptibility to severe DSS-induced colitis (Hashimoto et al., 2012).

Studies of several model animals have revealed that intestinal commensal flora affect host physiology and immunity. Although the mechanisms underlying this phenomenon have been partly 
revealed, e.g., acetic acid from $A$. pomorum modulates the host insulin pathway to support larval development, further analyses are needed to gain a complete mechanistic understanding of the effect of gut microbiota on the host. Furthermore, how hosts distinguish beneficial bacteria from pathogens remains mostly enigmatic. In Drosophila, the amount of uracil released from bacteria is the only factor so far identified to be involved in the bacterial discrimination. Notably, both acetic acid and uracil are small organic compounds. This seems to be reasonable: large molecules, such as cell wall components of bacteria, are commonly shared among bacteria phyla, making it difficult for hosts to distinguish the slight differences between bacterial species or to utilize them as a specific signal from microbes. Instead, bacterial metabolites, particularly secondary metabolites, are often restricted to a narrow set of species, suggesting that the hosts recognize those metabolites as a type of "bacterial signature." Many conventional studies on gut microbiota in association with nutrient intake and metabolism have examined the diversity and functions of small organic molecules. Investigators of innate immunology acknowledge the importance of bacterial "patterns" that are conserved within a class of microbes and sensed by pattern-recognition receptors. Future studies of gut immunology could target the diverse microbial metabolites as potential mediators of immune response and regulation.

\section{AUTHOR CONTRIBUTIONS}

Takayuki Kuraishi, Aki Hori, and Shoichiro Kurata wrote the paper.

\section{ACKNOWLEDGMENTS}

The authors apologize to colleagues whose work could not be directly cited due to space restrictions. We are grateful to Y. Kuraishi for preparing the figures. This work was supported by grants from the Japan Science and Technology Agency (JST); the Ministry of Education, Culture, Sports, Science and Technology of Japan (MEXT); the Kao Foundation for Arts and Sciences; and the Uehara Memorial Foundation.

\section{REFERENCES}

Abraham, E. G., and Jacobs-Lorena, M. (2004). Mosquito midgut barriers to malaria parasite development. Insect. Biochem. Mol. Biol. 34, 667-671. doi: 10.1016/j.ibmb.2004.03.019

Aggarwal, K., Rus, F., Vriesema-Magnuson, C., Ertürk-Hasdemir, D., Paquette, N., and Silverman, N. (2008). Rudra interrupts receptor signaling complexes to negatively regulate the IMD pathway. PLoS Pathog. 4:e1000120. doi: 10.1371/journal.ppat. 1000120

Apidianakis, Y., Pitsouli, C., Perrimon, N., and Rahme, L. (2009). Synergy between bacterial infection and genetic predisposition in intestinal dysplasia. Proc. Natl. Acad. Sci. U.S.A. 106, 20883-20888. doi: 10.1073/pnas.0911797106

Ayres, J. S., and Schneider, D. S. (2012). Tolerance of infections. Annu. Rev. Immunol. 30, 271-294. doi: 10.1146/annurev-immunol-020711-075030

Babior, B. M. (1999). NADPH oxidase: an update. Blood 93, 1464-1476.

Bae, Y. S., Choi, M. K., and Lee, W. J. (2010). Dual oxidase in mucosal immunity and host-microbe homeostasis. Trends Immunol. 31, 278-287. doi: 10.1016/j.it.2010.05.003

Basbous, N., Coste, F., Leone, P., Vincentelli, R., Royet, J., Kellenberger, C., et al. (2011). The Drosophila peptidoglycan-recognition protein LF interacts with peptidoglycan-recognition protein LC to downregulate the Imd pathway. EMBO Rep. 12, 327-333. doi: 10.1038/embor.2011.19
Bischoff, V., Vignal, C., Duvic, B., Boneca, I. G., Hoffmann, J. A., and Royet, J. (2006). Downregulation of the Drosophila immune response by peptidoglycanrecognition proteins SC1 and SC2. PLoS Pathog. 2:e14. doi: 10.1371/journal.ppat.0020014

Biteau, B., and Jasper, H. (2011). EGF signaling regulates the proliferation of intestinal stem cells in Drosophila. Development 138, 1045-1055. doi: 10.1242/dev.056671

Blemont, M., Vincentelli, R., Kellenberger, C., Opota, O., Lemaitre, B., Roussel, A., et al. (2013). Crystallization and preliminary X-ray analysis of monalysin, a novel-pore-forming toxin from the entomopathogen Pseudomonas entomophila. Acta Crystallogr. Sect. F Struct. Biol. Cryst. Commun. 69, 930-933. doi: 10.1107/S174430911301885X

Bond, D., and Foley, E. (2012). Autocrine PDGF-VEGF receptor-related (Pvr) pathway activity controls intestinal stem cell proliferation in the adult Drosophila midgut. J. Biol. Chem. 287, 27359-27370. doi: 10.1074/jbc.M112.378018

Bonnay, F., Cohen-Berros, E., Hoffmann, M., Kim, S. Y., Boulianne, G. L., Hoffmann, J. A., et al. (2013). big bang gene modulates gut immune tolerance in Drosophila. Proc. Natl. Acad. Sci. U.S.A. 110, 2957-2962. doi: 10.1073/pnas.1221910110

Bosco-Drayon, V., Poidevin, M., Boneca, I. G., Narbonne-Reveau, K., Royet, J., and Charroux, B. (2012). Peptidoglycan sensing by the receptor PGRP-LE in the Drosophila gut induces immune responses to infectious bacteria and tolerance to microbiota. Cell Host Microbe 12, 153-165. doi: 10.1016/j.chom.2012.06.002

Broderick, N. A., and Lemaitre, B. (2012). Gut-associated microbes of Drosophila melanogaster. Gut Microbes 3, 307-321. doi: 10.4161/gmic. 19896

Buchon, N., Broderick, N. A., Chakrabarti, S., and Lemaitre, B. (2009a). Invasive and indigenous microbiota impact intestinal stem cell activity through multiple pathways in Drosophila. Genes Dev. 23, 2333-2344. doi: 10.1101/gad.1827009

Buchon, N., Broderick, N. A., Poidevin, M., Pradervand, S., and Lemaitre, B. (2009b). Drosophila intestinal response to bacterial infection: activation of host defense and stem cell proliferation. Cell Host Microbe 5, 200-211. doi: 10.1016/j.chom.2009.01.003

Buchon, N., Broderick, N. A., Kuraishi, T., and Lemaitre, B. (2010). Drosophila EGFR pathway coordinates stem cell proliferation and gut remodeling following infection. BMC Biol. 8:152. doi: 10.1186/1741-7007-8-152

Buchon, N., Broderick, N. A., and Lemaitre, B. (2013a). Gut homeostasis in a microbial world: insights from Drosophila melanogaster. Nat. Rev. Microbiol. 11, 615-626. doi: 10.1038/nrmicro3074

Buchon, N., Osman, D., David, F. P. A., Boquete, J. P., Deplancke, B., and Lemaitre, B. (2013b). Morphological and molecular characterization of adult midgut compartmentalization in Drosophila. Cell Rep. 3, 1725-1738. doi: 10.1016/j.celrep.2013.04.001

Chakrabarti, S., Liehl, P., Buchon, N., and Lemaitre, B. (2012). Infectioninduced host translational blockage inhibits immune responses and epithelial renewal in the Drosophila gut. Cell Host Microbe 12, 60-70. doi: 10.1016/j.chom.2012.06.001

Chatterjee, M., and Ip, Y. T. (2009). Pathogenic stimulation of intestinal stem cell response in Drosophila. J. Cell. Physiol. 220, 664-671. doi: 10.1002/jcp.21808

Cirimotich, C. M., Dong, Y., Garver, L. S., Sim, S., and Dimopoulos, G. (2010). Mosquito immune defenses against Plasmodium infection. Dev. Comp. Immunol. 34, 387-395. doi: 10.1002/jcp.21808

Cognigni, P., Bailey, A. P., and Miguel-Aliaga, I. (2011). Enteric neurons and systemic signals couple nutritional and reproductive status with intestinal homeostasis. Cell Metab. 13, 92-104. doi: 10.1016/j.cmet.2010.12.010

Cordero, J. B., Stefanatos, R. K., Scopelliti, A., Vidal, M., and Sansom, O. J. (2012). Inducible progenitor-derived Wingless regulates adult midgut regeneration in Drosophila. EMBO J. 31, 3901-3917. doi: 10.1038/emboj.2012.248

Cronin, S. J., Nehme, N. T., Limmer, S., Liegeois, S., Pospisilik, J. A., Schramek, D., et al. (2009). Genome-wide RNAi screen identifies genes involved in intestinal pathogenic bacterial infection. Science 325, 340-343. doi: 10.1126/science. 1173164

Cummings, J. H., and MacFarlane, G. T. (1997). Role of intestinal bacteria in nutrient metabolism. JPEN J. Parenter. Enteral Nutr. 21, 357-365. doi: $10.1177 / 0148607197021006357$

Ferrandon, D. (2013). The complementary facets of epithelial host defenses in the genetic model organism Drosophila melanogaster: from resistance to resilience. Curr. Opin. Immunol. 25, 59-70. doi: 10.1016/j.coi.2012.11.008

Glittenberg, M. T., Kounatidis, I., Christensen, D., Kostov, M., Kimber, S., Roberts, I., et al. (2011). Pathogen and host factors are needed to provoke a systemic host 
response to gastrointestinal infection of Drosophila larvae by Candida albicans. Dis. Model. Mech. 4, 515-525. doi: 10.1242/dmm.006627

Ha, E. M., Lee, K. A., Park, S. H., Kim, S. H., Nam, H. J., Lee, H. Y., et al. (2009a). Regulation of DUOX by the Gaq-phospholipase C $\beta$ Ca2+ pathway in Drosophila gut immunity. Dev. Cell 16, 386-397. doi: 10.1016/j.devcel.2008.12.015

Ha, E. M., Lee, K. A., Seo, Y. Y., Kim, S. H., Lim, J. H., Oh, B. H., et al. (2009b). Coordination of multiple dual oxidase-regulatory pathways in responses to commensal and infectious microbes in drosophila gut. Nat. Immunol. 10, 949-957. doi: 10.1038/ni.1765

Ha, E. M., Oh, C. T., Bae, Y. S., and Lee, W. J. (2005). A direct role for dual oxidase in Drosophila gut immunity. Science 310, 847-850. doi: 10.1126/science.1117311

Hashimoto, T., Perlot, T., Rehman, A., Trichereau, J., Ishiguro, H., Paolino, M., et al. (2012). ACE2 links amino acid malnutrition to microbial ecology and intestinal inflammation. Nature 487, 477-481. doi: 10.1038/nature11228

Hayakawa, T., Shitomi, Y., Miyamoto, K., and Hori, H. (2004). GalNAc pretreatment inhibits trapping of Bacillus thuringiensis CrylAc on the peritrophic membrane of Bombyx mori. FEBS Lett. 576, 331-335. doi: 10.1016/j.febslet.2004.09.029

Hegan, P. S., Mermall, V., Tilney, L. G., and Mooseker, M. S. (2007). Roles for Drosophila melanogaster myosin IB in maintenance of enterocyte brush-border structure and resistance to the bacterial pathogen Pseudomonas entomophila. Mol. Biol. Cell 18, 4625-4636. doi: 10.1091/mbc.E07-02-0191

Hegedus, D., Erlandson, M., Gillott, C., and Toprak, U. (2009). New insights into peritrophic matrix synthesis, architecture, and function. Annu. Rev. Entomol. 54, 285-302. doi: 10.1146/annurev.ento.54.110807.090559

Iwasaki, A., and Medzhitov, R. (2010). Regulation of adaptive immunity by the innate immune system. Science 327, 291-295. doi: 10.1126/science.1183021

Jiang, H., Grenley, M. O., Bravo, M. J., Blumhagen, R. Z., and Edgar, B. A. (2011). EGFR/Ras/MAPK signaling mediates adult midgut epithelial homeostasis and regeneration in Drosophila. Cell Stem Cell 8, 84-95. doi: 10.1016/j.stem.2010.11.026

Jiang, H., Patel, P. H., Kohlmaier, A., Grenley, M. O., Mcewen, D. G., and Edgar, B. A. (2009). Cytokine/Jak/Stat signaling mediates regeneration and homeostasis in the Drosophila midgut. Cell 137, 1343-1355. doi: 10.1016/j.cell.2009.05.014

Karpowicz, P., Perez, J., and Perrimon, N. (2010). The Hippo tumor suppressor pathway regulates intestinal stem cell regeneration. Development 138, 4135-4145. doi: 10.1242/dev.060483

Kleino, A., Myllymäki, H., Kallio, J., Vanha-Aho, L. M., Oksanen, K., Ulvila, J., et al. (2008). Pirk is a negative regulator of the Drosophila Imd pathway. J. Immunol. 180, 5413-5422.

Kleino, A., and Silverman, N. (2013). The Drosophila IMD pathway in the activation of the humoral immune response. Dev. Comp. Immunol. 42, 25-35. doi: 10.1016/j.dci.2013.05.014

Kumar, S., Molina-Cruz, A., Gupta, L., Rodrigues, J., and Barillas-Mury, C. (2010). A peroxidase/dual oxidase system modulates midgut epithelial immunity in Anopheles gambiae. Science 327, 1644-1648. doi: 10.1126/science.1184008

Kuraishi, T., Binggeli, O., Opota, O., Buchon, N., and Lemaitre, B. (2011). Genetic evidence for a protective role of the peritrophic matrix against intestinal bacterial infection in Drosophila melanogaster. Proc. Natl. Acad. Sci. U.S.A. 108, 15966-15971. doi: 10.1073/pnas.1105994108

Kurata, S. (2004). Recognition of infectious non-self and activation of immune responses by peptidoglycan recognition protein (PGRP)-family members in Drosophila. Dev. Comp. Immunol. 28, 89-95. doi: 10.1016/S0145305X(03)00121-6

Kurata, S. (2010). Extracellular and intracellular pathogen recognition by Drosophila PGRP-LE and PGRP-LC. Int. Immunol. 22, 143-148. doi: 10.1093/intimm/dxp128

Lambrechts, L., Scott, T. W., and Gubler, D. J. (2010). Consequences of the expanding global distribution of Aedes albopictus for dengue virus transmission. PLoS Negl. Trop. Dis. 4:e646. doi: 10.1371/journal.pntd.0000646

Lee, K. A., Kim, S. H., Kim, E. K., Ha, E. M., You, H., Kim, B., et al. (2013). Bacterial-derived uracil as a modulator of mucosal immunity and gut-microbe homeostasis in Drosophila. Cell 153, 797-811. doi: 10.1016/J.Cell.2013.04.009

Lee, K. Z., and Ferrandon, D. (2011). Negative regulation of immune responses on the fly. EMBO J. 30, 988-990. doi: 10.1038/emboj.2011.47

Lehane, M. J. (1997). Peritrophic matrix structure and function. Annu. Rev. Entomol. 42, 525-550. doi: 10.1146/annurev.ento.42.1.525
Lemaitre, B., and Hoffmann, J. (2007). The host defense of Drosophila melanogaster. Annu. Rev. Immunol. 25, 697-743. doi: 10.1146/annurev.immunol.25.022106. 141615

Lemaitre, B., and Miguel-Aliaga, I. (2013). The digestive tract of Drosophila melanogaster. Annu. Rev. Genet. 47, 395-422. doi: 10.1146/annurev.genet. 111212-133343

Lhocine, N., Ribeiro, P. S., Buchon, N., Wepf, A., Wilson, R., Tenev, T., et al. (2008). PIMS modulates immune tolerance by negatively regulating Drosophila innate immune signaling. Cell Host Microbe 4, 147-158. doi: 10.1016/j.chom.2008.07.004

Liehl, P., Blight, M., Vodovar, N., Boccard, F., and Lemaitre, B. (2006). Prevalence of local immune response against oral infection in a Drosophila/Pseudomonas infection model. PLoS Pathog. 2:e56. doi: 10.1371/journal.ppat.0020056

Lin, G., Xu, N., and Xi, R. (2008). Paracrine Wingless signalling controls selfrenewal of Drosophila intestinal stem cells. Nature 455, 1119-1123. doi: 10.1038 /nature07329

Maillet, F., Bischoff, V., Vignal, C., Hoffmann, J., and Royet, J. (2008). The Drosophila peptidoglycan recognition protein PGRP-LF blocks PGRPLC and IMD/JNK pathway activation. Cell Host Microbe 3, 293-303. doi: 10.1016/j.chom.2008.04.002

Marianes, A., and Spradling, A. C. (2013). Physiological and stem cell compartmentalization within the Drosophila midgut. eLife 2, e00886. doi: 10.7554/eLife.00886

Micchelli, C. A., and Perrimon, N. (2006). Evidence that stem cells reside in the adult Drosophila midgut epithelium. Nature 439, 475-479. doi: 10.1038/nature04371

Nehme, N. T., Liégeois, S., Kele, B., Giammarinaro, P., Pradel, E., Hoffmann, J. A., et al. (2007). A model of bacterial intestinal infections in Drosophila melanogaster. PLoS Pathog. 3:e173. doi: 10.1371/journal.ppat.0030173

Neyen, C., Poidevin, M., Roussel, A., and Lemaitre, B. (2012). Tissue- and ligandspecific sensing of Gram-negative infection in Drosophila by PGRP-LC isoforms and PGRP-LE. J. Immunol. 189, 1886-1897. doi: 10.4049/jimmunol.1201022

Ohlstein, B., and Spradling, A. (2006). The adult Drosophila posterior midgut is maintained by pluripotent stem cells. Nature 439, 470-474. doi: 10.1038 /nature 04333

O’Neill, L. A., Golenbock, D., and Bowie, A. G. (2013). The history of Toll-like receptors - redefining innate immunity. Nat. Rev. Immunol. 13, 453-460. doi: $10.1038 /$ nri3446

Opota, O., Vallet-Gely, I., Vincentelli, R., Kellenberger, C., Iacovache, I., Gonzalez, M. R., et al. (2011). Monalysin, a novel $\beta$ - pore-forming toxin from the Drosophila pathogen Pseudomonas entomophila, contributes to host intestinal damage and lethality. PLoS Pathog. 7:e1002259. doi: 10.1371/journal.ppat.1002259

Osman, D., Buchon, N., Chakrabarti, S., Huang, Y. T., Su, W. C., Poidevin, M., et al. (2012). Autocrine and paracrine unpaired signaling regulate intestinal stem cell maintenance and division. J. Cell Sci. 125, 5944-5949. doi: 10.1242/ jcs. 113100

Paredes, J. C., Welchman, D. P., Poidevin, M., and Lemaitre, B. (2011). Negative regulation by amidase PGRPs shapes the Drosophila antibacterial response and protects the fly from innocuous infection. Immunity 35, 770-779. doi: 10.1016/j.immuni.2011.09.018

Ridley, E. V., Wong, A. C., Westmiller, S., and Douglas, A. E. (2012). Impact of the resident microbiota on the nutritional phenotype of Drosophila melanogaster. PLoS ONE 7:e36765. doi: 10.1371/journal.pone.0036765

Round, J. L., and Mazmanian, S. K. (2009). The gut microbiota shapes intestinal immune responses during health and disease. Nat. Rev. Immunol. 9, 313-323. doi: $10.1038 /$ nri2515

Royet, J. (2011). Epithelial homeostasis and the underlying molecular mechanisms in the gut of the insect model Drosophila melanogaster. Cell. Mol. Life Sci. 68, 3651-3660. doi: 10.1007/s00018-011-0828-x

Ryu, J. H., Ha, E. M., Oh, C. T., Seol, J. H., Brey, P. T., Jin, I., et al. (2006). An essential complementary role of NF- $\kappa \mathrm{B}$ pathway to microbicidal oxidants in Drosophila gut immunity. EMBO J 25, 3693-3701. doi: 10.1038/sj.emboj.7601233

Ryu, J. H., Kim, S. H., Lee, H. Y., Bai, J. Y., Nam, Y. D., Bae, J. W., et al. (2008). Innate immune homeostasis by the homeobox gene caudal and commensal-gut mutualism in Drosophila. Science 319, 777-782. doi: 10.1126/ science. 1149357 
Saha, S., Jing, X., Park, S. Y., Wang, S., Li, X., Gupta, D., et al. (2010). Peptidoglycan recognition proteins protect mice from experimental colitis by promoting normal gut flora and preventing induction of interferon- $\gamma$. Cell Host Microbe 8, 147-162. doi: 10.1016/j.chom.2010.07.005

Sartor, R. B. (2004). Microbial influences in inflammatory bowel diseases. Gastroenterology 134, 577-594. doi: 10.1053/j.gastro.2007.11.059

Shaw, R. L., Kohlmaier, A., Polesello, C., Veelken, C., Edgar, B. A., and Tapon, N. (2010). The Hippo pathway regulates intestinal stem cell proliferation during Drosophila adult midgut regeneration. Development 137, 4147-4158. doi: 10.1242/dev.052506

Shibata, T., Sekihara, S., Fujikawa, T., Miyaji, R., Maki, K., Ishihara, T., et al. (2013). Transglutaminase-catalyzed protein-protein cross-linking suppresses the activity of the NF-кB-like transcription factor Relish. Sci. Signal. 6, ra61. doi: 10.1126/scisignal.2003970

Shin, S. C., Kim, S. H., You, H., Kim, B., Kim, A. C., Lee, K. A., et al. (2011). Drosophila microbiome modulates host developmental and metabolic homeostasis via insulin signaling. Science 334, 670-674. doi: 10.1126/science.1212782

Staley, B. K., and Irvine, K. D. (2010). Warts and Yorkie mediate intestinal regeneration by influencing stem cell proliferation. Curr. Biol. 20, 1580-1587. doi: 10.1016/j.cub.2010.07.041

Storelli, G., Defaye, A., Erkosar, B., Hols, P., Royet, J., and Leulier, F. (2011). Lactobacillus plantarum promotes Drosophila systemic growth by modulating hormonal signals through TOR-dependent nutrient sensing. Cell Metab. 14, 403-414. doi: 10.1016/j.cmet.2011.07.012

Takeuchi, O., and Akira, S. (2010). Pattern recognition receptors and inflammation. Cell 140, 805-820. doi: 10.1016/j.cell.2010.01.022

Tellam, R. L. (1996). “The peritrophic matrix," in Biology of the Insect Midgut, ed M. J. Lehane and P. F. Billingsley (Cambridge: Chapman and Hall), 86-114.

Tzou, P., Ohresser, S., Ferrandon, D., Capovilla, M., Reichhart, J. M., Lemaitre, B., et al. (2000). Tissue-specific inducible expression of antimicrobial peptide genes in Drosophila surface epithelia. Immunity 13, 737-748. doi: 10.1016/S10747613(00)00072-8

Vodovar, N., Vinals, M., Liehl, P., Basset, A., Degrouard, J., Spellman, P., et al. (2005). Drosophila host defense after oral infection by an entomopathogenic Pseudomonas species. Proc. Natl. Acad. Sci. U.S.A. 102, 11414-11419. doi: 10.1073/pnas.0502240102
Wong, C. N. A., Ng, P., and Douglas, A. E. (2011). Low-diversity bacterial community in the gut of the fruitfly Drosophila melanogaster. Environ. Microbiol. 13, 1889-1900. doi: 10.1111/j.1462-2920.2011.02511.x

Yassine, H., and Osta, M. A. (2010). Anopheles gambiae innate immunity. Cell. Microbiol. 12, 1-9. doi: 10.1111/j.1462-5822.2009.01388.x

Yoshimoto, S., Loo, T. M., Atarashi, K., Kanda, H., Sato, S., Oyadomari, S., et al. (2013). Obesity-induced gut microbial metabolite promotes liver cancer through senescence secretome. Nature 499, 97-101. doi: 10.1038/ nature 12347

Zaidman-Rémy, A., Hervé, M., Poidevin, M., Pili-Floury, S., Kim, M. S., Blanot, D., et al. (2006). The Drosophila amidase PGRP-LB modulates the immune response to bacterial infection. Immunity 24, 463-473. doi: 10.1016/j.immuni.2006.02.012

Zasloff, M. (2002). Antimicrobial peptides of multicellular organisms. Nature 415, 389-395. doi: 10.1038/415389a

Zhou, F., Rasmussen, A., Lee, S., and Agaisse, H. (2013). The UPD3 cytokine couples environmental challenge and intestinal stem cell division through modulation of JAK/STAT signaling in the stem cell microenvironment. Dev. Biol. 373, 383-393. doi: 10.1016/j.ydbio.2012.10.023

Conflict of Interest Statement: The authors declare that the research was conducted in the absence of any commercial or financial relationships that could be construed as a potential conflict of interest.

Received: 27 October 2013; accepted: 02 December 2013; published online: 17 December 2013.

Citation: Kuraishi T, Hori A and Kurata S (2013) Host-microbe interactions in the gut of Drosophila melanogaster. Front. Physiol. 4:375. doi: 10.3389/fphys.2013.00375

This article was submitted to Integrative Physiology, a section of the journal Frontiers in Physiology.

Copyright (c) 2013 Kuraishi, Hori and Kurata. This is an open-access article distributed under the terms of the Creative Commons Attribution License (CC BY). The use, distribution or reproduction in other forums is permitted, provided the original author(s) or licensor are credited and that the original publication in this journal is cited, in accordance with accepted academic practice. No use, distribution or reproduction is permitted which does not comply with these terms. 